\title{
$\beta$-catenin/Tcf-4 complex transcriptionally regulates AKT1 in glioma
}

\author{
LINGCHAO CHEN $^{1 *}$, KAI HUANG ${ }^{2,3^{*}}$, LEI HAN ${ }^{2,3}$, ZHENDONG SHI $^{2,3}$, \\ KAILIANG ZHANG ${ }^{2,3}$, PEIYU PU ${ }^{2,3}$, CHUANLU JIANG ${ }^{1}$ and CHUNSHENG KANG ${ }^{2,3}$
}

\author{
${ }^{1}$ Department of Neurosurgery, the Second Affiliated Hospital of Harbin Medical University, Harbin 150086; \\ ${ }^{2}$ Department of Neurosurgery, Tianjin Medical University General Hospital, Laboratory of Neuro-Oncology, \\ Tianjin Neurological Institute; ${ }^{3}$ Key Laboratory of Neurotrauma, Variation and Regeneration, \\ Ministry of Education and Tianjin Municipal Government, Tianjin 300052, P.R.China
}

Received April 5, 2011; Accepted May 12, 2011

DOI: $10.3892 /$ ijo.2011.1104

\begin{abstract}
Increasing evidence suggests that interplays between Wnt/ $\beta$-catenin and PI3K/AKT signaling cascades are involved in tumor development and progression. However, the exact mechanism in glioma is not well known. Using aspirin, we found that the expression levels of AKT1 in glioma cells significantly correlated with the transcriptional activity of $\beta$-catenin. Similar observations were made when we subjected glioma cells to treatment with Tcf4 siRNA. Moreover, both aspirin and Tcf4 siRNA can suppress the proliferation and induce apoptosis of glioma. In addition, our analysis of the gene promoter of AKT1 revealed multiple putative Tcf-4 binding sites. In support of the concept that $\beta$-catenin/Tcf- 4 is a transcriptional regulator for AKT1, results from our chromatin immunoprecipitation studies and luciferase assay showed that $\beta$-catenin/Tcf- 4 binds to the potential binding sites in the gene promoter of AKT1. Furthermore, using immunohistochemistry, we found that Tcf-4 protein expression increased significantly in high-grade glioma in comparison to low-grade glioma and correlated with AKT1 expression. In conclusion, our results support the concept that $\beta$-catenin/Tcf-4 directly regulates AKT1 in glioma, and these two proteins may cooperate with each other in exerting their oncogenic effects in glioma.
\end{abstract}

\section{Introduction}

$\beta$-catenin is known to function as an adhesion molecule that is associated with E-cadherin and actin filaments at the cell membrane (1). In addition, it can act as a core factor involved in a

Correspondence to: Dr Chunsheng Kang, Laboratory of NeuroOncology, Tianjin Neurological Institute, 152 Ahshan Road, Heping, Tianjin 300052, P.R. China

E-mail:kang97061@yahoo.com

*Contributed equally

Key words: glioma, Tcf-4, AKT1, transcription number of cellular signaling pathways such as the Wnt canonical pathway and EGFR pathway (2). Further, $\beta$-catenin plays a pivotal role in mammary development and tumorigenesis through its functions in cell adhesion, signal transduction and regulation of cell-context-specific gene expression (3). As a cotranscripitonal factor, $\beta$-catenin was described to translocate into the nucleus. This nuclear translocation is regulated by several transcription factors of the TCF (T cell-factor) family such as Tcf-4 and Lef-1 (lymphoid-enhancer-factor-1), which were shown to interact with $\beta$-catenin $(4,5)$. The $\beta$-catenin/TCF complex recruits further chromatin-remodeling proteins to responsive promoters, thereby activating the transcription of specific target genes, including c-myc, fra-1 and cyclin D1 (6). Malignant gliomas are the most common primary brain tumors (7). Although $\beta$-catenin has been reported to be abnormally expressed in a variety of human cancers, little is known in gliomagenesis.

AKT, a serine/threonine kinase, is the essential mediator in the PI3K/AKT pathway which regulates the survival and apoptosis balance by different machnism $(8,9)$. Akt is also known to be involved in migration and invasion in cells of many different organisms (10). The combination of activated Ras and Akt induces high-grade gliomas with the histological features of human GBMs (11). Activated Akt1 prevents the phosphorylation and degradation of $\beta$-catenin by phosphorylating GSK-3 $\beta$ at ser9, which indicats the interplay between $\mathrm{Wnt} / \beta$-catenin and AKT signaling (12). Our previous data showed that downregulation of $\beta$-catenin by siRNA inhibites phosphorylation of AKT and suppresses malignant glioma cell growth (13). However, the definite mechanism between $\beta$-catenin/Tcf- 4 and AKT is not well explained. In this study, expression of the AKT1 was down-regulated both by aspirin and Tcf4 siRNA, leading us to the hypothesis that AKT1 might be regulated by $\beta$-catenin/Tcf- 4 . In addition, we established that transcription of AKT1 is indeed regulated by $\beta$-catenin/Tcf4 in glioma cells and overexpression of the AKT1 kinase co-localizes with high levels of Tcf4 in glioma samples.

\section{Patients and methods}

Patients and samples. Sixty glioma biopsies of fresh tumor tissue were obtained from patients operated at the Department 
of Neurosurgery at Tianjin Medical University General Hospital and classified according to WHO (World Health Organization) categories (2000). Tissues and clinical information are obtained as part of an approved study at the University. Pathologic grades of tumors were defined according to the 2000 WHO criteria, there are 15 cases in every grade. A portion of each tissue sample was snap-frozen in the liquid nitrogen following resection and stored at $-80^{\circ} \mathrm{C}$ for the remaining portion was fixed with $10 \%$ formalin for histopathological examination.

Immunohistochemistry. Serial formalin-fixed tissue sections of 60 glioma biopsies were stained using the Vectastain elite ABC detection system (Vector, Burlingame, CA, USA) according to the manufacturer's instructions. In brief, $2 \mathrm{~mm}$ sections were deparaffinized with xylene and passaged through decreasing concentrations of ethanol. Subsequently, antigen retrieval was performed by heating the slides in $10 \mathrm{mM}$ citric acid in a microwave oven (two times $5 \mathrm{~min}, 560 \mathrm{~W}$ ) at $\mathrm{pH} 6.0$. Tissue sections were incubated with first antibody (anti-AKT1, clone 2H10; diluted 1:100 in PBS or anti-Tcf-4 antibody diluted 1:200 in PBS) at $4^{\circ} \mathrm{C}$ overnight. After washing and incubation with secondary antibody (anti-mouse/anti-rabbit; Vector) for $30 \mathrm{~min}$, staining reaction was done using DakoCytomation AEC Chromogen (Dako GmbH, Hamburg, Germany) as substrate.

Cell culture, transfection and chemicals. Human glioblastoma cell lines (LN229, U87, SNB19) were obtained from China Academia Sinica cell repository, Shanghai, China. The cells were maintained in Dulbecco's modified Eagle's medium (DMEM, Gibco) supplemented with 10\% fetal bovine serum, and incubated at $37^{\circ} \mathrm{C}$ with $5 \% \mathrm{CO}_{2}$. Tcf-4 siRNA and nonsense siRNA were both synthesized by Shanghai Genepharma Co. Inc. (Shanghai, China). Tcf-4 siRNA directed sequence: 5'-TTCTCCGAACGTGTCACGT-3'. Nonsense siRNA directed sequence: 5'-AGACGAGGGCGAACAGGAG-3'. The vectors expressing human $\beta$-catenin and Tcf- 4 were kind gifts of Professor Jinquan Cheng (H. Lee Moffitt Cancer Center and Research Institute, USA) and the recombinant adenoviruses Ad.CMV- $\beta$-catenin and Ad.CMV-Tcf-4 recombinant adenovirus were generated using the Adxsi system (Sinogenomax Co., Ltd., Beijing, China). Antisense AKT1 plasmids pLXSN-As-AKT1 were also kindly provided by Professor Jinquan Cheng. Aspirin (acetylsalicylic acid) was purchased from Sigma. A 1 M stock solution was prepared in acetone. Cells were carried out in the presence or the absence of increasing concentrations $(0.5,1,5,10$ or $20 \mathrm{mM}$ ) of aspirin for $1,2,6,12$ or $24 \mathrm{~h}$.

Apoptosis. Forty-eight hours after transfection, apoptosis in cultured cells was evaluated with annexin $\mathrm{V}$ labeling, caspase 3/7 activity and mitochondrial membrane potential. For the annexin $\mathrm{V}$ assay, an annexin V-FITC labeled Apoptosis Detection Kit (Abcam) was used according to the manufacturer's protocol.

Cell viability assay. The cell viability was determined by the MTT (3-(4, 5-dimethylthiazole)-2, 5-diphenyltetrazoliumbromide) assay. Briefly, LN229 and U87 cells were seeded into 96-well plates at 4,000 cells per well and allowed to attach overnight. On each day of consecutive 5 days after treatment, MTT solution $(5 \mathrm{mg} / \mathrm{ml})$ was added to each well and the cells were incubated at $37^{\circ} \mathrm{C}$ for $4 \mathrm{~h}$. The reaction was then stopped by lysing. Optical density (OD) was measured at the wavelength of $450 \mathrm{~nm}$. The data are presented as the mean \pm standard deviation (SD), which are derived from triplicate samples of at least three independent experiments.

ChIP assay. ChIP analysis was done using a ChIP assay kit (Upstate Biotechnology) according to the manufacturer's instructions. LN229 cell was used, and immunoprecipitation was done overnight at $4^{\circ} \mathrm{C}$ with $10 \mu \mathrm{l}$ of the sample used as the 'input' or $1 \mu \mathrm{g}$ of anti-Tcf-4 (Upstate) or the negative control mouse IgG, positive control anti-RNA polymerase beads. After reverse cross-linking, DNA was purified with QIAquick PCR purification kit (Qiagen, Valencia, CA, USA) from the immunoprecipitates. PCR was carried out with 35 cycles of $95^{\circ} \mathrm{C}$ for $30 \mathrm{sec}, 61^{\circ} \mathrm{C}$ for $45 \mathrm{sec}$, and $72^{\circ} \mathrm{C}$ for $45 \mathrm{sec}$ using the following primers flanking the putative Tcf-4-binding sites in the human AKT1 promoter. Binding site 1: upper: 5'-AGGCCTTGGAGGGGCTCAGG-3'; lower: 5'-CCTCAGGCACAGGGGGCTCT-3'. Binding site 2: upper: 5'-CAGCAGCAGGCCTTGGAGGG-3'; lower: 5'-CA CTGAGTGGGCGTGGCCTG-3'. Binding site 3 and 4: upper: 5'-CAGCAGCAGGCCTTGGAGGG-3'; lower: 5'-CCTCAGG CACAGGGGGCTCT-3'. Binding site 5: upper: 5'-GTGAGGG TTGGCTGGTGGGC-3'; lower:5'-CAGTGCTGGGCGCTGG AGAC-3'. Binding sites 6 and 7: upper: 5'-GTCCTGCGGTGC CTTCCTCG -3'; lower: 5'-AGGCAAGGGCTGGACACC CA-3'. Binding site 8: upper: 5'-TCGGAGTCCTGCGGTGCC TT-3'; lower: 5'- GGCAAGGGCTGGACACCCAC-3'.

Western blot analysis. Prepared cells were lysed in $1 \%$ Nonidet P-40 lysis buffer after treatment. Homogenates were clarified by centrifugation at $20,000 \mathrm{x}$ g for $15 \mathrm{~min}$ at $4^{\circ} \mathrm{C}$, and protein concentration was measured by the Lowry method. SDS-PAGE was performed on $40 \mu \mathrm{g}$ of protein from each sample, gels were transferred to PVDF membranes (Millipore, USA) and incubated with primary antibodies against AKT1 (Cell Signaling Technology), Fra-1 (Santa Cruz Biotechnology), Tcf-4 (Santa Cruz Biotechnology), $\beta$-catenin (Cell Signaling Technology), and GAPDH, followed by incubation with HRP-conjugated secondary antibody (1:1000 dilution, Zymed, San Diego, CA). After washing with stripping buffer, the membrane was reprobed with antibody against GAPDH.

Luciferase reporter assay. To evaluate the $\beta$-catenin/Tcf- 4 transcriptional activity, we used a pair of luciferase reporterconstructs, TOP-FLASH and FOP-FLASH (Upstate). TOP-FLASH (with three repeats of the Tcf-binding site) or FOP-FLASH (with three repeats of a mutated Tcf-binding site) plasmids were transfected into cells treated with aspirin, as instructed by the suppliers. Luciferase activity was measured with the Dual-luciferase reporter assay system, with the Renilla luciferase activity as an internal control, $48 \mathrm{~h}$ after transfection. In addition, pGL3-WT6AKT1-promoter and pGL3-MUT6-AKT1-promoter reporters were were kindly provided by professor Susanne Dihlmann.

Subcutaneous tumor assay. LN229 glioma cells were subcutaneously injected to 5-week old female nude mice (Cancer Institute of The Chinese Academy of Medical Science). When the tumor volume reached $50 \mathrm{~mm}^{3}$, the mice were randomly divided into three groups (ten mice per group) which were treated with nonsense siRNA $(50 \mathrm{nmol} / \mathrm{l} \times 10 \mu \mathrm{l})$ mixture, the control group 


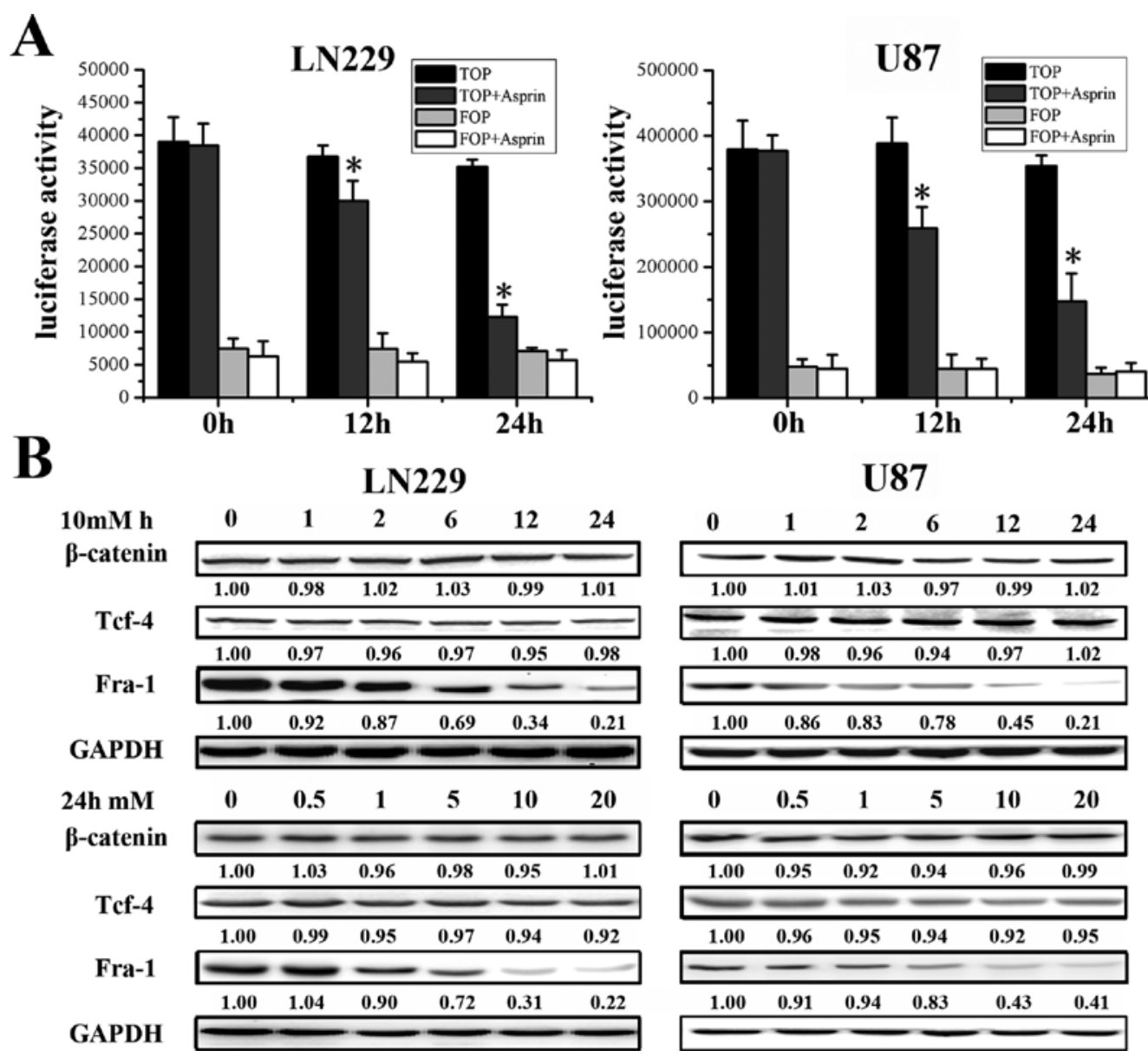

Figure 1. Aspirin down-regulates $\beta$-catenin activity in glioma cells. (A) Twenty-four hours after transfected with TOP or FOP luciferase reporter constructs, the cells were treated with $10 \mathrm{mM}$ aspirin for the indicated times. $\beta$-catenin/Tcf-4-dependent transcription was analyzed by measuring luciferase activity. Inhibition of $\beta$-catenin/Tcf-4-dependent transcription in response to aspirin treatment was the strongest at $24 \mathrm{~h}$ time-point both in LN229 and U87cells. (B) The cells treated with aspirin were carried out in the presence or the absence of increasing concentrations and time-points, $\beta$-catenin, Tcf- 4 and Fra- 1 expression was observed by Western blotting. The quantitative data under the bands show the relative protein levels (normalized to GAPDH level) in the control group.

(20 $\mu \mathrm{l}$ PBS) and Tcf-4 si group (a mixture of $20 \mu \mathrm{l}$ Lipofectamine and Tcf-4 siRNA) local injection of xenograft tumor in multiple sites. The treatment was performed once every 2 days for 20 days. and the tumor volume was measured with a caliper every 2 days, using the formula volume $=$ length $\times$ width $^{2} / 2$. At the end of 20-day observation period, the mice bearing xenograft tumors were sacrificed and the tumor tissues were removed for formalin fixation and preparation of paraffin embedded sections for immunohistochemical analysis.

Statistical analysis. Data were analyzed with SPSS 10.0. ANOVA, t-test, $\chi^{2}$ test and Pearson's correlation were used to analyze the significance between groups. Statistical significance was assigned to P-values $<0.05$.

\section{Results}

Aspirin down-regulates $\beta$-catenin activity in glioma cells. Previous work showed that transcription of $\mathrm{Wnt} / \beta$-catenin target genes is inhibited by aspirin and indomethacin in CRC cells (14). To determine whether this effect happens in gliomas, parallel samples of the LN229 and U87 cells were transiently transfected with a $\beta$-catenin/TCF-responsive luciferase reporter construct. In LN229 cells, aspirin reduced Topflash activity in a time-dependent manner at a concentration of $10 \mathrm{mM}, 20 \%$ and $60 \%$ of Topflash activity was inhibited respectively in 6 and $24 \mathrm{~h}$ compared with untreated cells. U87 cells showed a similar result. Western blot showed the total amount of $\beta$-catenin and Tcf- 4 were not decreased either in a time- or dose-dependent manner. Furthermore, one of the most important targets of $\beta$-catenin, Fra-1, showed reduced expression after the treatment by aspirin. Thus, aspirin down-regulates $\beta$-catenin activity in glioma cells in a time- and concentration-dependent manner (Fig. 1).

Effect of $\beta$-catenin/Tcf-4 activity on glioma cell proliferation and apoptosis. To detect the biological effect of low $\beta$-catenin/ Tcf-4 transcriptional activity on glioma cell proliferation, MTT and Annexin V-PI assay were used. As shown in Fig. 2C, inhibition of $\beta$-catenin/Tcf- 4 with asprin suppressed the proliferation of U87 and LN229 cells, beginning $24 \mathrm{~h}$ after administration and continuing throughout the 6-day observation period, as determined by MTT assay (Fig. 2A). In contrast, acetone did not impact U87 and LN229 cell proliferation. As observed in the Annexin V-PI assay, the apoptosis percentage of control, acetone and aspirin group in LN229 cells was 3.16, 3.23 and $15.9 \%$, respectively. Similar trend of apoptosis rate was detected in U87 cells. These results suggest that $\beta$-catenin/Tcf- 4 activity is critical for glioma cell proliferation and apoptosis. 


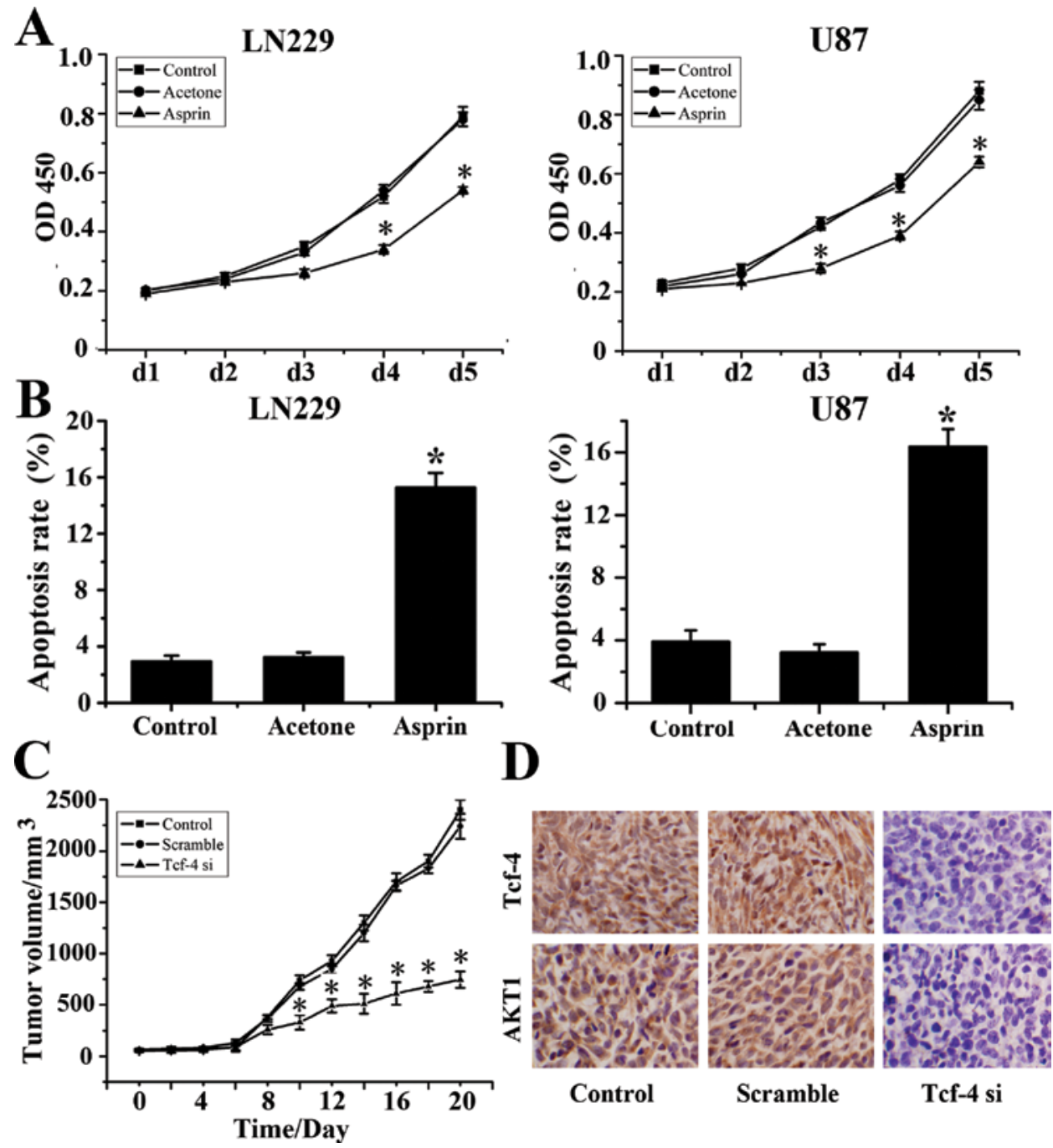

Figure 2. Effect of $\beta$-catenin/Tcf-4 activity on glioma cell and glioma xenograft growth. (A) Aspirin induced apoptosis in LN229 and U87 cell lines. The cells were treated with $10 \mathrm{mM}$ aspirin for $72 \mathrm{~h}$, and subjected to Annexin-PI analyses. (B) LN229 and U87 cells survival was quantified by MTT assay for 5 days following treatment. Data represent the mean \pm SD. (C) When subcutaneous tumors were established, Tcf-4 siRNA was injected in a multi-site injection manner. Tumor volumes were measured every 2 days during treatment. Down-regulation of Tcf- 4 expression efficiently decreased tumor growth in vivo. "P<0.05 compared with the control group. (D) Immunohistochemical analysis of the expression of AKT1 and Tcf-4 28 days following Tcf-4 si administrations. Data are from one of three representative experiments.

Inhibition of Tcf-4 suppresses glioma xenograft growth and impacts on protein expression. To explore the effect of $\beta$-catenin/ Tcf-4 activity on tumor growth in vivo, we employed a xenograft LN229 glioma mouse model treated with Tcf-4 siRNA. On day 8 , the tumors of Tcf- 4 si group started to reach statistical significance compared with control groups $(\mathrm{P}<0.05)$. At the termination of the study, the difference in tumor mass between Tcf4 group and control groups was marked (Fig. 6). After mice were observed for 20 days, the tumor samples were taken out, and paraffin-embedded section was prepared for immunohistopathological examination. In the course of analyzing the effect of Tcf- 4 siRNA we observed that the protein level of Tcf-4 and AKT1 were similarly down-regulated as compared to those treated with scrambled siRNA.

The expression of AKT1 positively correlates with $\beta$-catenin/Tcf-4 activity. Next, we want to investigate whether the expression of AKT1 correlated with $\beta$-catenin/Tcf-4 in vitro. As anticipated, AKT1 was similarly down-regulated as well as $\beta$-catenin/Tcf-4 target gene Fra-1 treated by asprin. Moreover, using Tcf-4 siRNA, we identified a significant reduction of AKT1 expression and phosphorylation of AKT. SNB 19 cells, which have low $\beta$-catenin and Tcf-4 expression, were transfected with Ad. $\beta$-catenin and/or Ad.Tcf-4 recombinant adenovirus. Overexpression of $\beta$-catenin and Tcf-4 in SNB19 cells induced an increase of AKT1 expression and phosphorylation of AKT. Therefore, we hypothesized that AKT1 expression might be a direct target of $\beta$-catenin/Tcf-4 complex in glioma cells (Fig. 3).

$\beta$-catenin/Tcf-4 target AKT1 promoter regions. To specify the functional interaction between $\beta$-catenin/Tcf- 4 signaling and AKT1 gene expression, we scanned the AKT1 gene promoter region for regulating DNA-binding elements. A search of the GenBank nucleotide database revealed the presence of 8 putative $\beta$-catenin/Tcf-4-binding sites in the AKT1 gene, showing a high degree of homology to the core consensus sequence (CTTTG or CAAAG). To establish in vivo binding of $\beta$-catenin/ Tcf- 4 to the AKT1 promoter we performed ChIP assays in 


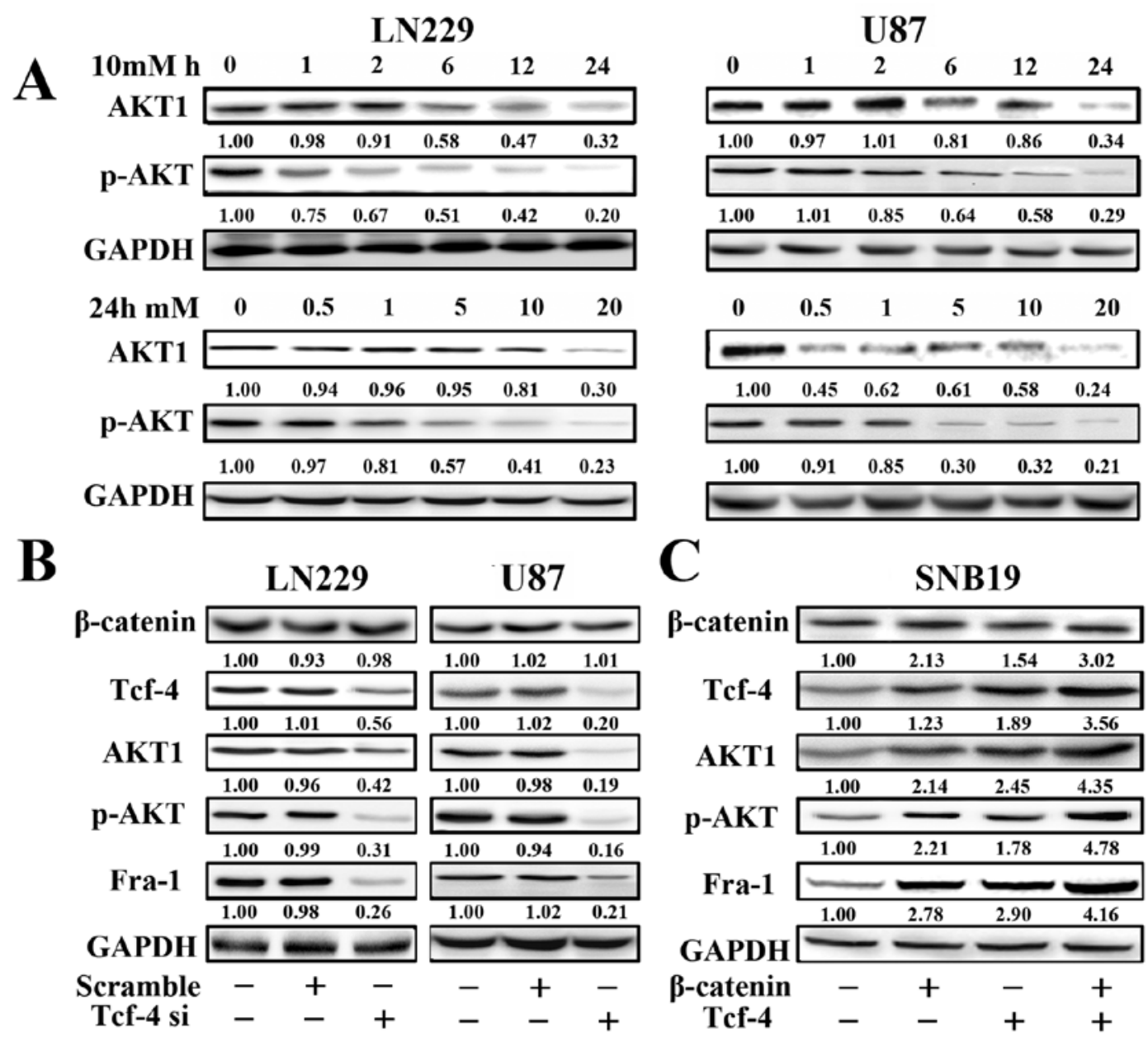

Figure 3. AKT1 expression is regulated by $\beta$-catenin/Tcf-4 activity. (A) The cells treated with aspirin were studied in the presence or the absence of increasing concentrations and various time-points. Western blotting showed a clear reduction of AKT1 expression and phosphorylation of AKT in a time- and dose-dependent manner. (B) Tcf-4 down-regulation by siRNA triggered a significant repression of AKT1 expression in LN229 and U87 cells. (C) Overexpression of $\beta$-catenin and/or Tcf-4 induced an increase of AKT1 expression. The quantitative data under the bands show the relative protein levels (normalized to GAPDH level) in the control group. Data are presented as the means of triplicate experiments.

LN229 cells. Six primers were designed to amplify the putative $\beta$-catenin/Tcf-4-binding sites in our study. As shown in Fig. 4, only primer 4 which targets binding site 6 showed amplifiable products. In contrast, no detectable amplification was observed for other primers. The input lanes were included as a control for the PCR effectiveness. PCR without the addition of DNA templates was used as a negative control.

To test the specificity of the TBE site 6 for transactivation by $\beta$-catenin/Tcf-4, TBE6 mutant and wild-type AKT1 promoter luciferase plasmids were transiently transfected into LN229 and U87 cells. Wild-type AKT1 promoter demonstrated a low activity after reduction of Tcf-4 and TBE6 mutant AKT1 promoter could abolish the inhibitory effect of Tcf- 4 siRNA on luciferase activity. Thus, the putative TBE site 6 exhibits promoter activative function in glioma cells. Taken together, these results strongly support the hypothesis that $\beta$-catenin/Tcf- 4 contribute to the regulation of AKT1 expression.

Tcf-4 and AKT1 expression positively correlate with glioma grades. Using immunohistochemistry, we surveyed the expression of Tcf4 and AKT1 in cohort of formalin-fixed, paraffin-embedded glioma samples. The results showed that Tcf-4 and AKT1 expression is positively correlated with glioma grades. Heat map representing gene expression level is shown in Fig. 5. Pearson's correlation showed that a significant positive correlation existed between Tcf- 4 and AKT1 expression $(\mathrm{R}=0.845, \mathrm{P}<0.001)$ (Fig. 5B). Accordingly, Tcf-4 expression increases significantly with the ascending pathologic grade of gliomas and correlates positively with AKT1 expression.

Reduction of AKT1 inhibits glioma cell proliferation and induces apoptosis. To investigate the biological importance of AKT1 on glioma, we down-regulated AKT1 levels in LN229 and U87 using siRNA, and a significant down-regulation of AKT1 was shown on Western blotting (Fig. 6A). The Annexin-PI analysis showed that apoptosisi was induced both in LN229 and U87 cells used by AKT1 si (Fig. 6B). In addtion, MTT assay was also performed. As shown in Fig. 6C, there was a significant decrease in cell growth in cells treated with AKT1 siRNA, as compared to those treated with scrambled siRNA. The results suggest that glioma cell proliferation regulated by $\beta$-catenin/Tcf- 4 activity is, in part, mediated by AKT1. 

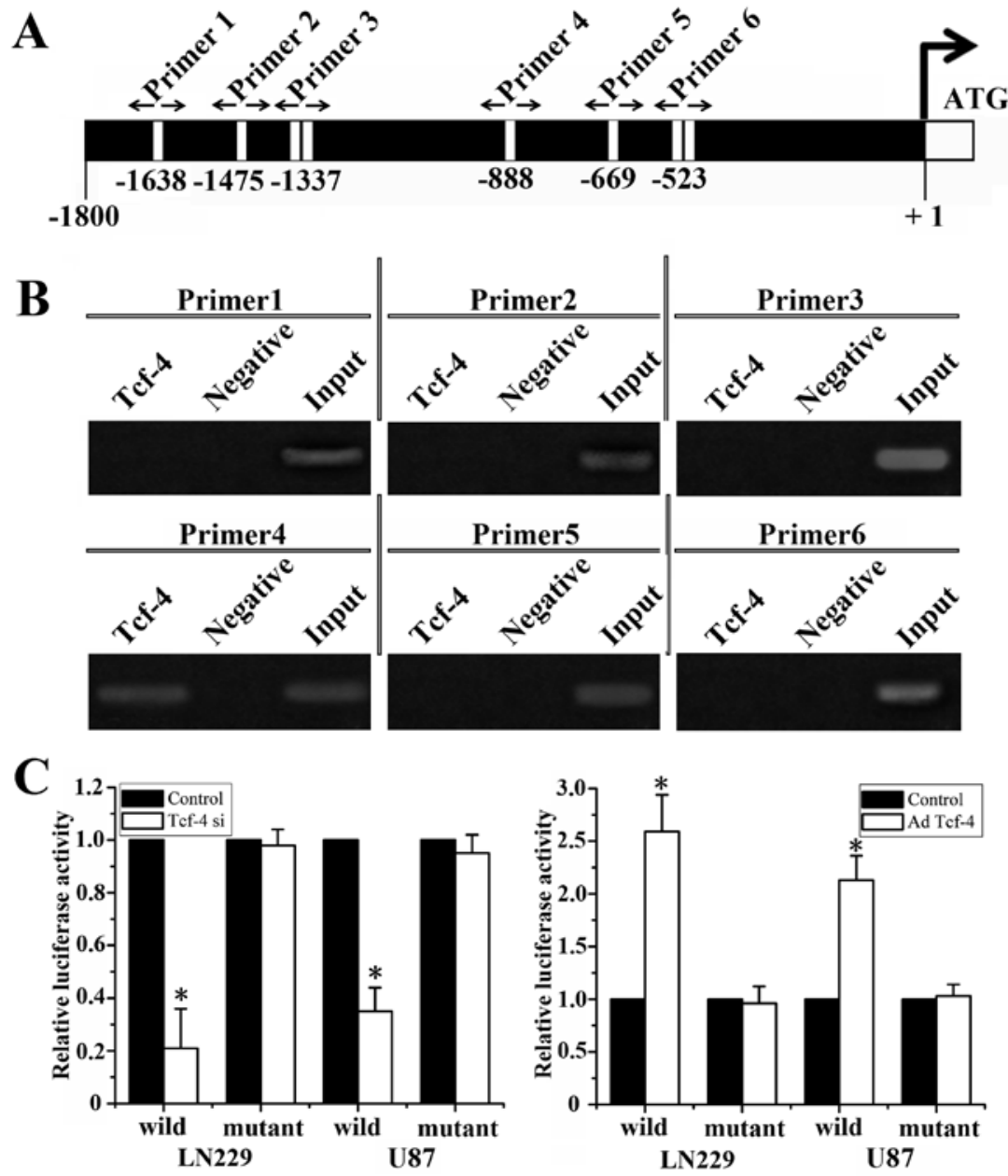

Figure 4. AKT1 is a direct target of $\beta$-catenin/Tcf-4. (A) The promoter region of the AKT1 gene was analyzed for consensus $\beta$-catenin/Tcf-4-binding sites. Diagram of the AKT1 promoter with location of $\beta$-catenin/Tcf-4-binding sites is indicated. (B) Chromatin immunoprecipitation was performed using LN229 cells. A rabbit polyclonal antibody against Tcf- 4 was used. Normal rabbit IgG antibody served as a negative control. PCR with primer 4 revealed amplifications. In contrast, no amplifications were detected with other primers and the negative control. (C) Effects of Tcf-4 si and Ad.Tcf-4 on the activity of the AKT1 promoter/ enhancer in LN229 and U87 cells. Mutations in the Tcf/Lef binding sites reduce the activation of the AKT1 promoter/enhancer. LN229 cells were cotransfected with the indicated reporter constructs and Ad.Tcf-4. Bars indicate SD of two independent experiments.

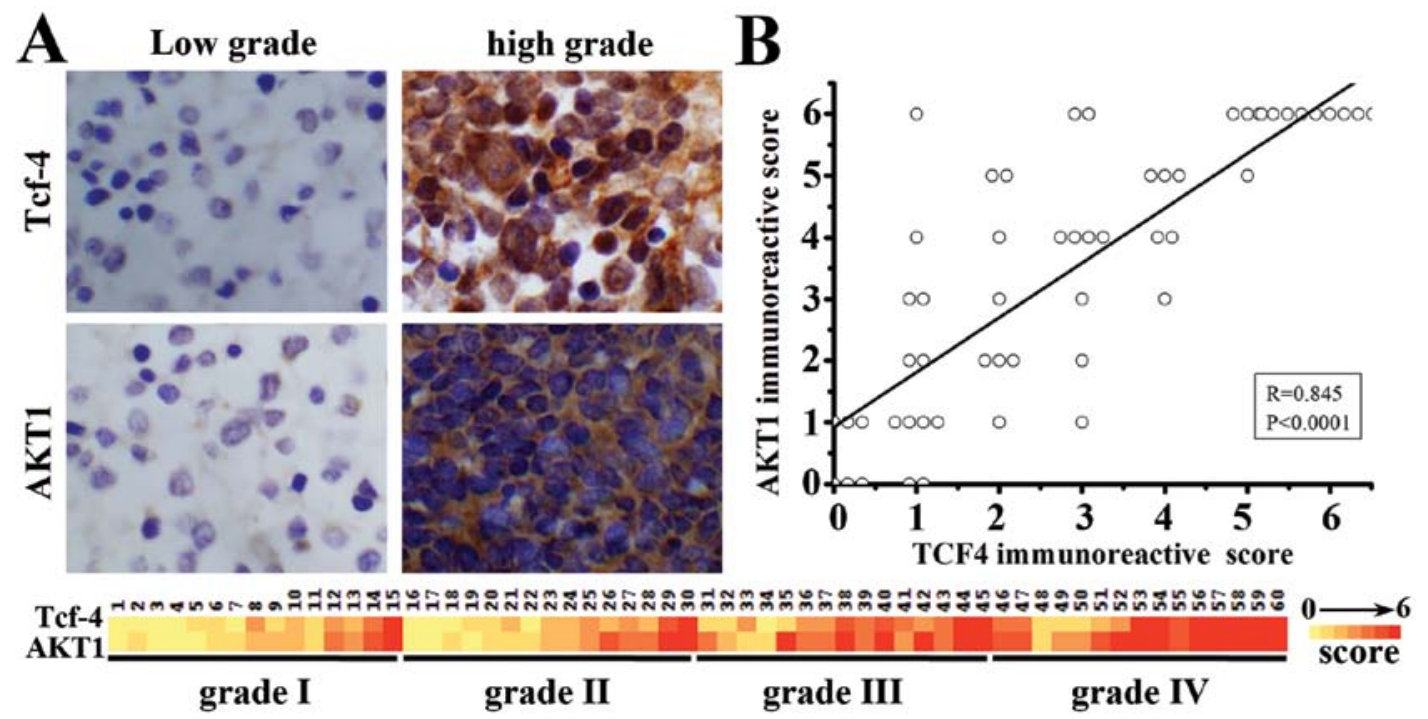

Figure 5. Expression of Tcf-4 and AKT1 in glioma. The expression of Tcf-4 and AKT1 was detected in glioma tissues by immunostaining. The levels of Tcf-4 and AKT1 protein increased markedly in high grade gliomas (WHO grade III and IV) in comparison to low grade gliomas (WHO grade I and II). Heat map representing gene expression level is determined in a linear scale with a maximum value of 6 immunoreactive score to highlight differences within this range. (B) Positive correlation of Tcf-4 expression with AKT1 expression by Pearson correlation analysis. A trend line is provided in each plot, which represents the 'best fit' as determined by simple linear regression. 
A

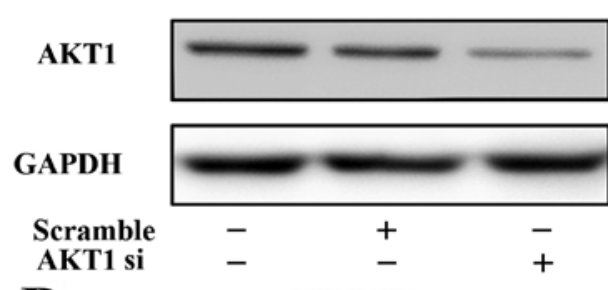

U87
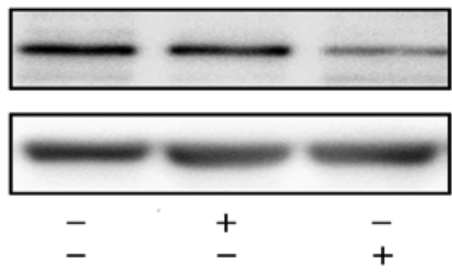

U87
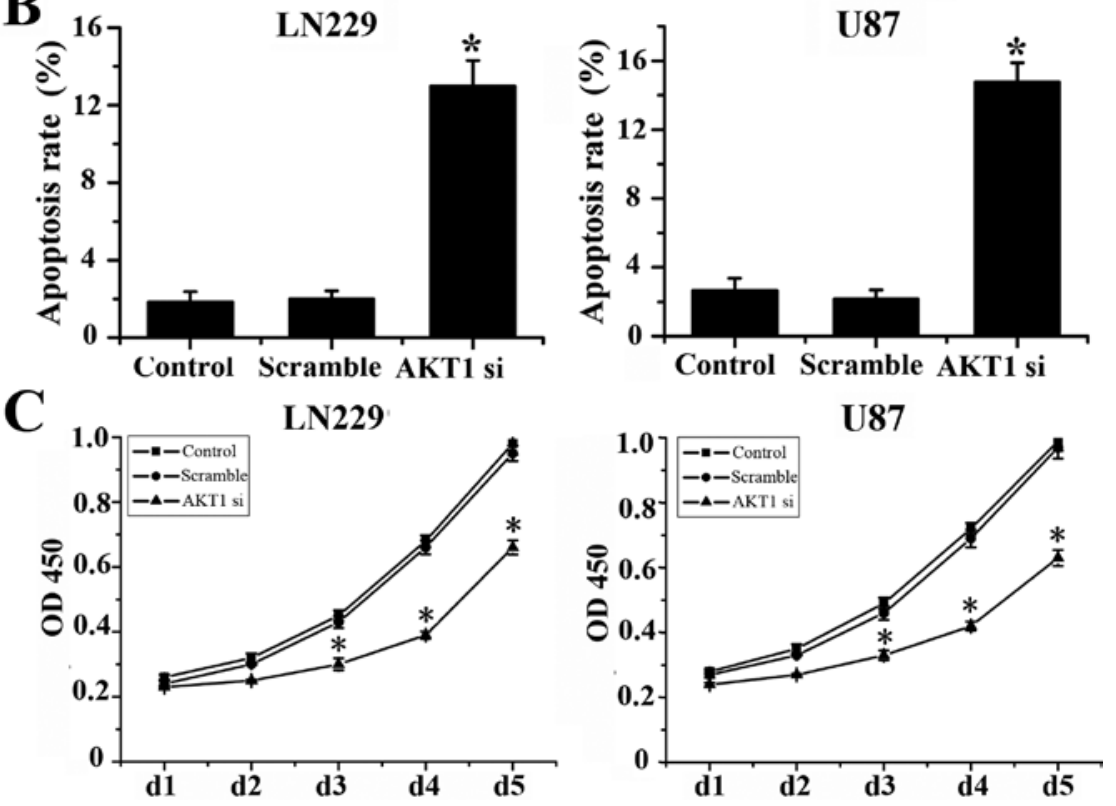

Figure 6. Reduction of AKT1 decreases glioma cell proliferation and induces apoptosis. (A) LN229 and U87 cells were transfected with antisense AKT1, and AKT1 expression was detected by Western blot assay. GAPDH was regarded as endogenous normalizer. (B) Reduction of AKT1 induced apoptosis rate both in U87 and LN229. The cells were treated with antisense AKT1 for $72 \mathrm{~h}$, and subjected to Annexin-PI analyses by FACS. (C) LN229 and U87 cell survival was quantified by MTT assay for 5 days following AKT1 si treatment. Data represent the mean \pm SD.

\section{Discussion}

AKT, a serine/threonine kinase, also known as PKB, consists of three members: Akt1/PKB $\alpha, \mathrm{Akt} 2 / \mathrm{PKB} \beta$, and Akt3/PKB $\gamma$. All Akt family members share similar tructure with an $\mathrm{N}$-terminal plekstrin homology domain and a C-terminal hydrophobic domain (15). In order to be fully activated, Akt requires phosphorylation at two specific amino acid residues, threonine 308 and serine 473 , respectively. Upon activation, Akt moves to the cytoplasm and nucleus where it phosphorylates downstream target proteins, including mTOR, FKHR, GSK-3, mdm2, IкB IKK, and BAD (16), ultimately affecting a number of processes containing cell growth, survival, proliferation, metabolism, and motility and/or invasion. As we know, oncogenic activation of the PI3K signalling pathway plays a pivotal role in the development of GBM (17). A central node in PI3K downstream signalling is controlled by the serine-threonine kinase AKT1. Previous data demonstrated that shRNA targeting Akt1 down-regulates its expression significantly in a sequence-specific manner, exerting proliferation and invasion inhibition effects on U251 cells (18). In our study, immunohistochemistry assay showed that AKT1 expression is positively correlated with glioma grades. Reduction of AKT1 by siRNA significantly inhibits glioma cells proliferation and induces cell cycle arrest. Our results emphasize the importance of AKT1 in high grade glioma. $\beta$-catenin and Tcf- 4 are the core components of the canonical Wnt/ $\beta$-catenin/Tcf pathway (5). Upon translocation to the nucleus, $\beta$-catenin interacts with Tcf4 and Lef1, constituting a complex which binds to an evolutionarily conserved consensus motif on the promoter of target genes, including c-myc, cyclin D and Fra-1. In addition, plenty of new targets have recently been identified in the website of Stanford university (http://www. stanford.edu/ rnusse/pathways/targets.html). Multitude of target genes are known to play a pivotal role in controlling growth, proliferation of cells (19-21). Recently, the Cancer Genome Atlas Network showed that the Proneural, one of the molecular subtype of glioblastoma, contains several signatures of proneural development genes, such as SOX and Tcf-4 (22). Additionally, Proneural subtype of glioblastoma was considered of no benefit in the response to aggressive therapy. Our present study showed that reduction of Tcf- 4 can decrease the expression of AKT1. Importantly, knockdown of Tcf-4 suppresses the proliferation of glioma both in vivo and in vitro. Accrodingly, these data suggest that Tcf-4 may play an important role in glioma progression and prognosis or prediction of response to therapy.

Our previous study demostrated that knockdown of $\beta$-catenin inhibited growth of glioma cells and down-regulated the expression of PI3K/AKT pathway, indicating the interplay between Wnt/ $\beta$-catenin and AKT signaling cascades. Moreover, Liu et al, recently reported that $\mathrm{Wnt} / \beta$-catenin pathway corre- 
lated closely with the progression of gliomas and might be a novel prognostic marker for this neoplasm. In literature, most of dowmstream genes were directly regulated by $\beta$-catenin/Tcf were reported in colorect cancer (23-25). Few studies reported the transcript regulation of $\beta$-catenin/Tcf in glioma. Here, we establish that down-regulation of $\beta$-catenin/Tcf activity induces glioma cell apoptosis, and suppresses the proliferation of glioma. These effects are mediated in part by the direct transcriptional regulation of the oncogenic AKT1. We demonstrate that Tcf-4 directly targets the promoter regions of AKT1 and induces the transcription of AKT1 by CHIP assay and luciferase assay respectively. Furthermore, knockdown of AKT1 results in a similar effect compared to treatment with Tcf-4 siRNA.

In conclusion, our present studies establish that $\beta$-catenin/ Tcf-4 activity regulates AKT1 expression by binding to the AKT1 promoter, thereby modulating glioma cell growth. In addition, we indicate that Tcf- 4 expression increases significantly with the ascending pathologic grade of gliomas and correlates positively with AKT1 expression. Since specific agents inhibiting $\beta$-catenin/Tcf-4 and AKT1 are available $(26,27)$, our data provide the rationale for combining these inhibitors in treating specific forms of cancer, such as in a subset of glioma in which both $\beta$-catenin/Tcf- 4 and AKT1 are activated.

\section{Acknowledgements}

This work was supported by China National Natural Scientific Fund (30971136), China National Natural Scientific Fund (81001128), Tianjin City High School Science \& Technology Fund (2009CD01). The authors wish to thank Dr Susanne Dihlmann, Institute of Molecular Pathology, University Hospital Heidelberg, for kindly providing the wild- and mutant-type of AKT1 promoter reporter plasmids.

\section{References}

1. Hirohashi S and Kanai Y: Cell adhesion system and human cancer morphogenesis. Cancer Sci 94: 575-581, 2003.

2. Schlange T, Matsuda Y, Lienhard S, Huber A and Hynes NE: Autocrine WNT signaling contributes to breast cancer cell proliferation via the canonical WNT pathway and EGFR transactivation. Breast Cancer Res 9: R63, 2007.

3. Incassati A, Chandramouli A, Eelkema R and Cowin P: Key signaling nodes in mammary gland development and cancer: beta-catenin. Breast Cancer Res 12: 213, 2010.

4. Kimelman $\mathrm{D}$ and $\mathrm{Xu} \mathrm{W}$ : Beta-catenin destruction complex: insights and questions from a structural perspective. Oncogene 25: 7482-7491, 2006.

5. Huang K, Zhang JX, Han L, You YP, Jiang T, Pu PY, et al: MicroRNA roles in beta-catenin pathway. Mol Cancer 9: 252, 2010.

6. Tetsu O and McCormick F: Beta-catenin regulates expression of cyclin D1 in colon carcinoma cells. Nature 398: 422-426, 1999.

7. Huang TT, Sarkaria SM, Cloughesy TF and Mischel PS: Targeted therapy for malignant glioma patients: lessons learned and the road ahead. Neurotherapeutics 6: 500-512, 2009.

8. Bleeker FE, Lamba S, Zanon C, van Tilborg AA, Leenstra S, Troost D, et al: Absence of AKT1 mutations in glioblastoma PLoS One 4: e5638, 2009.
9. Franke TF, Hornik CP, Segev L, Shostak GA and Sugimoto C: PI3K/Akt and apoptosis: size matters. Oncogene 22: 8983-8998, 2003.

10. Manning BD and Cantley LC: AKT/PKB signaling: navigating downstream. Cell 129: 1261-1274, 2007.

11. Holland EC, Celestino J, Dai C, Schaefer L, Sawaya RE and Fuller GN: Combined activation of Ras and Akt in neural progenitors induces glioblastoma formation in mice. Nat Genet 25: 55-57, 2000.

12. Korkaya H, Paulson A, Charafe-Jauffret E, Ginestier C, Brown M, Dutcher J, et al: Regulation of mammary stem/progenitor cells by PTEN/Akt/beta-catenin signaling. PLoS Biol 7: e1000121, 2009.

13. Pu P, Zhang Z, Kang C, Jiang R, Jia Z, Wang G, et al: Downregulation of Wnt 2 and beta-catenin by siRNA suppresses malignant glioma cell growth. Cancer Gene Ther 16: 351-361, 2009.

14. Dihlmann S, Siermann A and von Knebel Doeberitz M: The nonsteroidal anti-inflammatory drugs aspirin and indomethacin attenuate beta-catenin/TCF-4 signaling. Oncogene 20: 645-653, 2001.

15. Dillon RL and Muller WJ: Distinct biological roles for the Akt family in mammary tumor progression. Cancer Res 70: 4260-4264, 2010.

16. West KA, Castillo SS and Dennis PA: Activation of the PI3K/Akt pathway and chemotherapeutic resistance. Drug Resist Updat 5: 234-248, 2002.

17. Mischel PS and Cloughesy TF: Targeted molecular therapy of GBM. Brain Pathol 13: 52-61, 2003.

18. Zhang J, Zhang QY, Fu YC, Wang T, Xu P, Zhou X, et al: Expression of p-Akt and COX-2 in gastric adenocarcinomas and adenovirus mediated Akt1 and COX-2 ShRNA suppresses SGC-7901 gastric adenocarcinoma and U251 glioma cell growth in vitro and in vivo. Technol Cancer Res Treat 8: 467-478, 2009.

19. Brabletz T, Jung A, Dag S, Hlubek F and Kirchner T: beta-catenin regulates the expression of the matrix metalloproteinase-7 in human colorectal cancer. Am J Pathol 155: 1033-1038, 1999.

20. Rodilla V, Villanueva A, Obrador-Hevia A, Robert-Moreno A, Fernandez-Majada V, Grilli A, et al: Jaggedl is the pathological link between Wnt and Notch pathways in colorectal cancer. Proc Natl Acad Sci USA 106: 6315-6320, 2009.

21. Mann B, Gelos M, Siedow A, Hanski ML, Gratchev A, Ilyas M, et al: Target genes of beta-catenin-T cell-factor/lymphoid-enhancerfactor signaling in human colorectal carcinomas. Proc Natl Acad Sci USA 96: 1603-1608, 1999.

22. Verhaak RG,Hoadley KA,PurdomE, Wang V, Qi Y, Wilkerson MD, et al: Integrated genomic analysis identifies clinically relevant subtypes of glioblastoma characterized by abnormalities in PDGFRA, IDH1, EGFR, and NF1. Cancer Cell 17: 98-110, 2010.

23. Lapham A, Adams JE, Paterson A, Lee M, Brimmell M and Packham G: The Bcl-w promoter is activated by beta-catenin/ TCF4 in human colorectal carcinoma cells. Gene 432: 112-117, 2009.

24. Yochum GS, Cleland R and Goodman RH: A genome-wide screen for beta-catenin binding sites identifies a downstream enhancer element that controls c-Myc gene expression. Mol Cell Biol 28: 7368-7379, 2008

25. Shimokawa T, Furukawa Y, Sakai M, Li M, Miwa N, Lin YM, et al: Involvement of the FGF18 gene in colorectal carcinogenesis, as a novel downstream target of the beta-catenin/T-cell factor complex. Cancer Res 63: 6116-6120, 2003.

26. Handeli S and Simon JA: A small-molecule inhibitor of Tcf/betacatenin signaling down-regulates PPARgamma and PPARdelta activities. Mol Cancer Ther 7: 521-529, 2008.

27. Wu WI, Voegtli WC, Sturgis HL, Dizon FP, Vigers GP and Brandhuber BJ: Crystal structure of human AKT1 with an allosteric inhibitor reveals a new mode of kinase inhibition. PLoS One 5: e12913, 2010. 